\title{
Effect of elemental diet combined with infliximab dose escalation in patients with Crohn's disease with loss of response to infliximab: CERISIER trial
}

Tadakazu Hisamatsu ${ }^{1 *}$, Reiko Kunisaki ${ }^{2 *}$, Shiro Nakamura ${ }^{3}$, Tomoyuki Tsujikawa ${ }^{4}$, Fumihito Hirai ${ }^{5}$, Hiroshi Nakase ${ }^{6}$, Kenji Watanabe ${ }^{7,8}$, Kaoru Yokoyama ${ }^{9}$, Masakazu Nagahori ${ }^{10}$, Takanori Kanai ${ }^{11}$, Makoto Naganuma $^{11}$, Hirofumi Michimae ${ }^{12}$, Akira Andoh ${ }^{13}$, Akihiro Yamada ${ }^{14}$, Tadashi Yokoyama ${ }^{15}$, Noriko Kamata $^{16}$, Shinji Tanaka ${ }^{17}$, Yasuo Suzuki ${ }^{14}$, Toshifumi Hibi ${ }^{18}$, Mamoru Watanabe ${ }^{10}$, CERISIER Trial group ${ }^{\#}$

${ }^{1}$ The Third Department of Internal Medicine, Kyorin University School of Medicine, Mitaka, ${ }^{2}$ Inflammatory Bowel Disease Center, Yokohama City University Medical Center, Yokohama, ${ }^{3}$ Department of Inflammatory Bowel Disease, Hyogo College of Medicine, Nishinomiya, ${ }^{4}$ Department of Gastroenterology and Hepatology, National Hospital Organization, Higashi-Ohmi Medical Center, Higashi-Ohmi, ${ }^{5}$ Department of Gastroenterology, Fukuoka University Chikushi Hospital, Chikushino, ${ }^{6}$ Department of Gastroenterology and Hepatology, Sapporo Medical University School of Medicine, Sapporo, ${ }^{7}$ Division of Gastroenterology, Osaka City General Hospital, Osaka, ${ }^{8}$ Department of Intestinal Inflammation Research, Hyogo College of Medicine, Nishinomiya, ${ }^{9}$ Department of Gastroenterology, Kitasato University School of Medicine, Sagamihara, ${ }^{10}$ Department of Gastroenterology and Hepatology, Tokyo Medical and Dental University, Tokyo, ${ }^{11}$ Division of Gastroenterology and Hepatology, Department of Internal Medicine, Keio University School of Medicine, Tokyo, ${ }^{12}$ Department of Clinical Medicine (Biostatistics), Kitasato University School of Pharmacy, Tokyo, ${ }^{13}$ Division of Gastroenterology, Shiga University of Medical Science, Otsu, ${ }^{14}$ Department of Internal Medicine, Toho University Sakura Medical Centre, Sakura, ${ }^{15}$ Yokoyama IBD Clinic, Aichi, ${ }^{16}$ Department of Gastroenterology, Osaka City University Graduate School of Medicine, Osaka, ${ }^{17}$ Department of Endoscopy, Hiroshima University Hospital, Hiroshima, ${ }^{18}$ Center for Advanced IBD Research and Treatment, Kitasato University Kitasato Institute Hospital, Tokyo, Japan

The development and success of anti-tumor necrosis factor- $\alpha$ (anti-TNF- $\alpha$ ) monoclonal antibodies (mAbs) have dramatically changed the therapeutic strategy of IBD and

Received October 23, 2017. Revised December 29, 2017.

Accepted January 9, 2018.

Correspondence to Tadakazu Hisamatsu, Division of Gastroenterology and Hepatology, The Third Department of Internal Medicine, Kyorin University School of Medicine, 6-20-2 Shinkawa, Mitaka 181-8611, Japan. Tel: +81422-47-5511 (ext. 5729), Fax: +81-422-71-5381, E-mail: thisamatsu@ ks.kyorin-u.ac.jp

*These authors contributed equally to this study.

"Combination of Elemental Dietary Therapy and Infliximab for Secondary Failure to Infliximab in Patients with Crohn's Disease (CERISIER) Trial group members: Hideaki Kimura ${ }^{2}$, Shingo Kato ${ }^{19}$, Shigeki Bamba ${ }^{13}$, Yuji Naito ${ }^{20}$, Takuya Inoue ${ }^{21}$, Shuhei Hosomi ${ }^{16}$, Masaki limuro ${ }^{3}$, Akira Harada ${ }^{22}$, Takahiro Beppu $^{5}$

${ }^{19}$ Department of Gastroenterology and Hepatology, Saitama Medical Centre, Saitama Medical University, Kawagoe, ${ }^{20}$ Department of Molecular Gastroenterology and Hepatology, Graduate School of Medical Science, Kyoto Prefectural University of Medicine, Kyoto, ${ }^{21} 2$ nd Department of Internal Medicine, Osaka Medical College, Takatsuki, ${ }^{22}$ Division of Gastroenterology, Matsuyama Red Cross Hospital, Matsuyama, Japan contributed to improvements in patients' quality of life. ${ }^{1}$ Many clinical observations have confirmed that anti-TNF- $\alpha$ mAbs are efficacious for the treatment of CD. Unfortunately, however, we are faced with a new complication: loss of response (LOR). In Japan at the time of this study, an infliximab (IFX) dose escalation regimen to $10 \mathrm{mg} / \mathrm{kg}$ was only allowed in patients who developed LOR during scheduled maintenance therapy with IFX ( $5 \mathrm{mg} / \mathrm{kg}$ every 8 weeks) according to the results of a preapproval clinical trial. However, we often encounter patients who cannot gain adequate control with increasing doses of $10 \mathrm{mg} / \mathrm{kg}$ of IFX in daily clinical practice. In this open-labeled prospective study (UMIN registration No. 000010058), patients with non-colonic CD who had developed LOR to scheduled administration of IFX ( $5 \mathrm{mg} / \mathrm{kg}$ every 8 weeks) were randomly assigned to IFX dose escalation ( $10 \mathrm{mg} / \mathrm{kg}$ every 8 weeks) with (combination group) or without (monotherapy group) an elemental diet (ED, 900-1200 kcal/day) for 56 weeks (Table 1, Supple-

\footnotetext{
๑ Copyright 2018. Korean Association for the Study of Intestinal Diseases. All rights reserved.

This is an Open Access article distributed under the terms of the Creative Commons Attribution Non-Commercial License (http://creativecommons.org/licenses/by-nc/4.0)

which permits unrestricted non-commercial use, distribution, and reproduction in any medium, provided the original work is properly cited.
} 
Table 1. Baseline Characteristics

\begin{tabular}{|c|c|c|c|}
\hline & $\begin{array}{c}\text { IFX mono } \\
\text { group }(n=6)\end{array}$ & $\begin{array}{l}\text { ED+IFX group } \\
(n=14)\end{array}$ & $P$-value \\
\hline \multicolumn{4}{|l|}{ Demographics } \\
\hline Sex (male/female) & $5 / 1$ & $11 / 3$ & 1.0000 \\
\hline Age (yr) & $35.20 \pm 10.11$ & $34.80 \pm 8.81$ & 0.9202 \\
\hline BMI $\left(\mathrm{kg} / \mathrm{m}^{2}\right)$ & $21.40 \pm 4.54$ & $21.00 \pm 4.32$ & 0.9044 \\
\hline CDAl score & $210.70 \pm 17.47$ & $211.00 \pm 60.78$ & 0.1256 \\
\hline Disease duration & $9.24 \pm 7.23$ & $11.13 \pm 9.04$ & 0.9680 \\
\hline Disease location & & & 1.0000 \\
\hline Ileitis (L1) & $1(16.7)$ & $3(21.4)$ & \\
\hline Ileocolitis (L2) & $5(83.3)$ & $11(78.6)$ & \\
\hline Perianal lesion & & & 1.0000 \\
\hline Yes & $2(33.3)$ & 4 (28.6) & \\
\hline No & $4(66.7)$ & $10(71.4)$ & \\
\hline \multicolumn{2}{|c|}{ Previous surgical resections } & & 1.0000 \\
\hline 0 & $3(50.0)$ & $6(42.9)$ & \\
\hline$\geq 1$ & $3(50.0)$ & $8(57.1)$ & \\
\hline Current smoking & $1(16.7)$ & $2(14.3)$ & 1.0000 \\
\hline \multicolumn{4}{|l|}{ Medication at entry } \\
\hline Immunomodulator & $2(33.3)$ & $4(28.6)$ & 1.0000 \\
\hline Steroid use & 0 & 0 & - \\
\hline 5-ASA & 5 (83.3) & 12 (85.7) & 1.0000 \\
\hline \multicolumn{4}{|c|}{ Concomitant medication } \\
\hline Immunomodulator & 2 (33.3) & $5(35.7)$ & 1.0000 \\
\hline Steroid use & 1 (16.7) & 0 & 0.3000 \\
\hline 5-ASA & 5 (83.3) & 12 (85.7) & 1.0000 \\
\hline $\mathrm{CRP}(\mathrm{mg} / \mathrm{dL})$ & $0.34 \pm 0.26$ & $0.74 \pm 0.81$ & 0.5466 \\
\hline Serum albumin (g/dL) & $3.88 \pm 0.32$ & $3.91 \pm 0.63$ & 0.4268 \\
\hline
\end{tabular}

For calculation of the $P$-value, Wilcoxon's exact test was used for continuous values and Fisher exact test was used for categorical values. Statistical analyses were performed at a significance level of 0.05 (twosided).

IFX, infliximab; ED, elemental diet; 5-ASA, 5-aminosalicylic acid.

mentary Figs. 1 and 2). The study was approved by the Institutional Review Board of Keio University Hospital (IRB No. 20120438) and performed in accordance with the principles of the Declaration of Helsinki. The informed consent was written informed consents were obtained. The primary endpoint was the retention rate of IFX dose escalation therapy at week 56. Safety, the CDAI, and the serum albumin and CRP levels at each observation point were analyzed. Intermediate analysis was performed on 15 patients enrolled by 31 March 2015. At week 16, 8 of 11 patients in the combination group and 0 of 4 patients in the monotherapy group showed a CDAI reduction from baseline $(\Delta \mathrm{CDAI})$ of $\geq 50$. The intermediate analysis showed the benefits of combination therapy and disadvantage of monotherapy, and the study was stopped. Subsequent analysis showed that the proportion of patients with $\triangle \mathrm{CDAI}$ of $\geq 50$ (Fig. 1A) and clinical remission (Fig. 1B) at each observation point tended to be higher in the combination group. Compared with the monotherapy group, the combination group showed a tendency toward a superior persistence rate of IFX ( $10 \mathrm{mg} / \mathrm{kg}$ every 8 weeks) at week 56 as the primary endpoint in both the per-protocol set analysis $(P=0.1066)$ and full analysis set analysis $(P=0.1629)$ (Fig. 2). No serious adverse events were observed in either group (Supplementary Table 1). This appears to be the first clinical trial to show the usefulness of combination therapy of enteral nutrition (EN) therapy with biologics for refractory CD.

The effectiveness of EN therapy in patients with CD, especially for the maintenance of clinical remission, has been previously reported..$^{2-4}$ Several recent reports have described combination therapy involving anti-TNF- $\alpha$ mAb therapy with EN therapy. Hirai et al. ${ }^{5}$ examined 102 patients with CD who were treated with IFX. The cumulative remission rate was significantly higher in the combination therapy group (IFX+EN) than in the non-EN group. Kamata et al. ${ }^{6}$ reported that concomitant ED therapy $(\geq 900 \mathrm{kcal} /$ day) with IFX scheduled maintenance therapy prevented LOR. Sazuka et al. ${ }^{7}$ also reported the benefit of concomitant use of EN therapy ( $\geq 600 \mathrm{kcal} /$ day) with IFX scheduled maintenance therapy. Sugita et al. ${ }^{8}$ reported that ED therapy administered concomitantly with adalimumab reduced LOR to adalimumab in IFX-intolerant or IFX-refractory patients. Nguyen et al. ${ }^{9}$ performed a meta-analysis based on these reports and concluded that in patients with moderate to severe CD undergoing IFX therapy, combined EN therapy of $\geq 600 \mathrm{kcal} /$ day affected the increase in the remission maintenance rate. In contrast, Yamamoto et al. ${ }^{10}$ reported the results of a prospective study showing that concomitant EN during IFX maintenance therapy did not show a beneficial effect in the maintenance rate of clinical remission in patients with $\mathrm{CD}$. However, in the EN combination group, the CDAI tended to be lower at each observation time point than in the IFX alone group. In the study by Yamamoto et al., ${ }^{10}$ the patients with CD had been newly introduced to IFX. These findings may suggest that IFX and EN combination therapy should be considered in only selected patients, including those with suspected LOR to IFX.

There are several limitations in this study. First, we discon- 
A

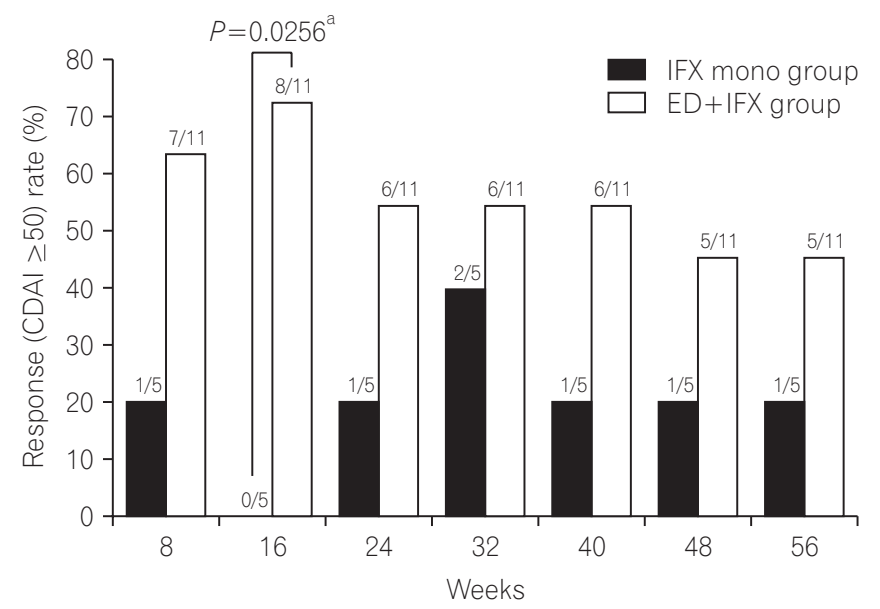

B

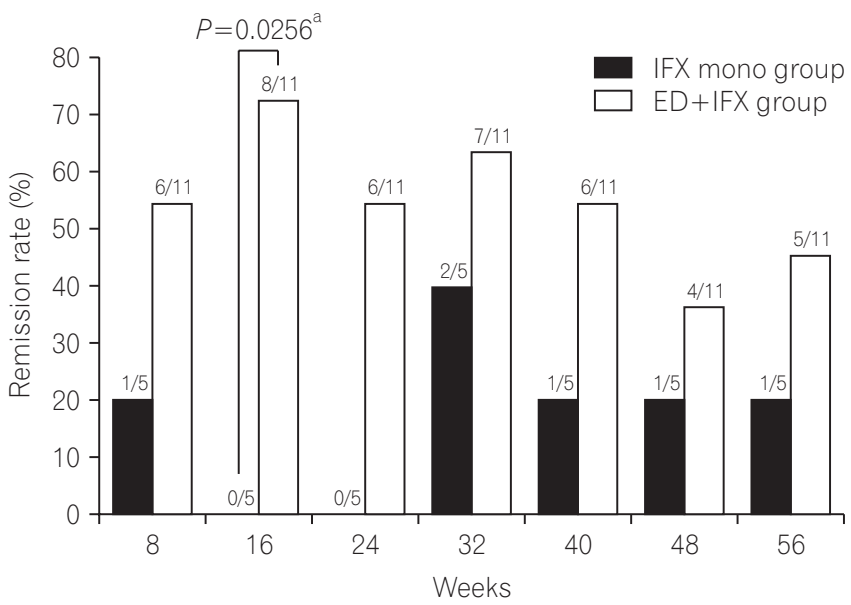

Fig. 1. Percentage of patients with a decrease in CDAl from weeks 8 to 56 . (A) The percentage of patients who satisfied the $\triangle C D A l$ of $\geq 50$ from weeks 8 to 56 of treatment is shown. Proportions of patients were compared between the groups by Fisher's exact test. Statistical analyses were performed at a two-sided significance level of 0.05. (B) The percentage of patients who achieved clinical remission, as indicated by a CDAl of $<150$, from weeks 8 to 56 of treatment is shown. Proportions of patients were compared between the groups by Fisher's exact test. Statistical analyses were performed at a two-sided significance level of 0.05 . ${ }^{a}$ Significant difference. ED, elemental diet; IFX, infliximab.

A

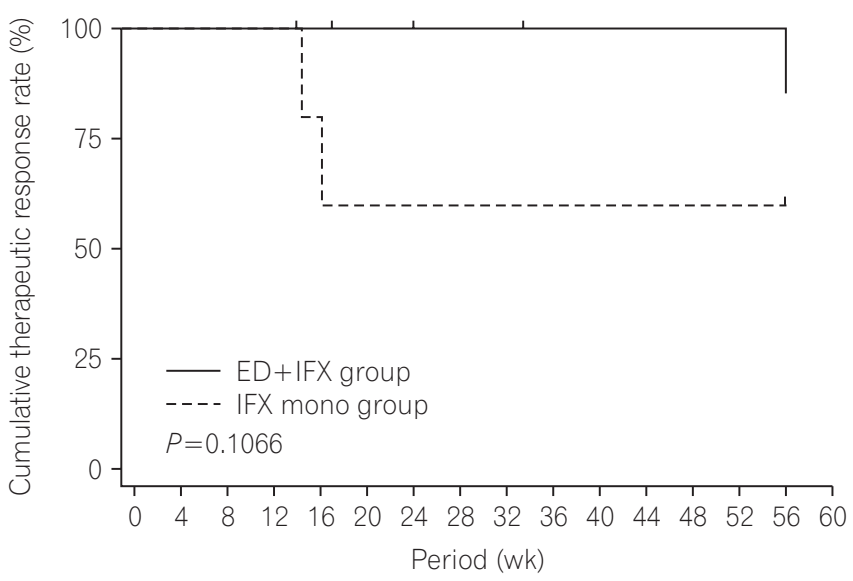

B

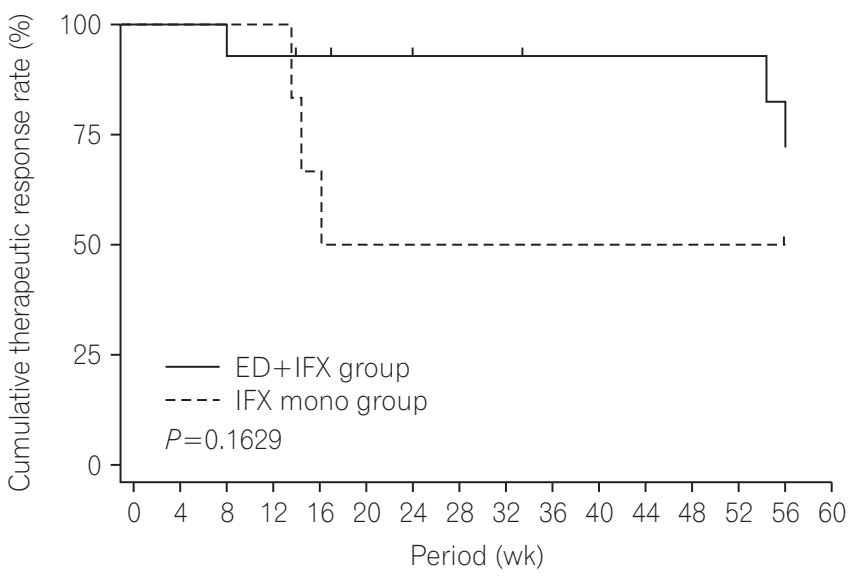

Fig. 2. Cumulative rate of successful completion of the scheduled maintenance treatment with IFX infusion at 10 mg/kg every 8 weeks until week 56 . (A) The scheduled maintenance treatment continuation rate curve was estimated by the Kaplan-Meier method for each group, and the groups were compared by the generalized Wilcoxon test (per-protocol set). (B) The scheduled maintenance treatment continuation rate curve was estimated by the Kaplan-Meier method for each group, and the groups were compared by the generalized Wilcoxon test (full analysis set). IFX, infliximab; ED, elemental diet.

tinued this study after obtaining the results of the interim analysis, which actually resulted in statistical underpowering of this investigation. Second, the protocol of this study did not include evaluation of endoscopic activity. Third, maintenance of the relatively high acceptance rate of taking ED in the combination group was influenced by the enroll- ment of only patients who had appropriate adherence to $\mathrm{ED}$, as judged by the acceptability test before enrollment and the patients' history. Therefore, in daily clinical practice, adherence to oral ED administration is expected to be lower. Improvement of adherence to therapy is an important point when using ED to treat CD. 


\section{FINANCIAL SUPPORT}

This work was funded by EA Pharma Co., Ltd. and undertaken by the Japanese Society for Inflammatory Bowel Disease.

\section{CONFLICT OF INTEREST}

This work was funded by EA Pharma Co., Ltd.

\section{AUTHOR CONTRIBUTION}

Study concept and design: Tadakazu Hisamatsu, Reiko Kunisaki, Shiro Nakamura, Tomoyuki Tsujikawa, Fumihito Hirai, Hiroshi Nakase, Kenji Watanabe, Kaoru Yokoyama, Masakazu Nagahori

Acquisition of data: Tadakazu Hisamatsu, Reiko Kunisaki, Hideaki Kimura, Shiro Nakamura, Fumihito Hirai, Kenji Watanabe, Makoto Naganuma, Takanori Kanai, Akihiro Yamada, Yasuo Suzuki, Noriko Kamata, Tadashi Yokoyama, Shinji Tanaka, Shingo Kato, Shigeki Bamba, Akira Andoh, Yuji Naito, Takuya Inoue, Shuhei Hosomi, Masaki Iimuro, Akira Harada, Takahiro Beppu

Analysis and interpretation of data: Tadakazu Hisamatsu, Reiko Kunisaki, Shiro Nakamura, Tomoyuki Tsujikawa, Fumihito Hirai, Hiroshi Nakase, Kenji Watanabe, Kaoru Yokoyama, Masakazu Nagahori, Akira Andoh

Drafting of the manuscript: Tadakazu Hisamatsu, Toshifumi Hibi

Critical revision of the manuscript for important intellectual content: Yasuo Suzuki, Mamoru Watanabe, Toshifumi Hibi

Statistical analysis: Hirofumi Michimae

Study supervision: Yasuo Suzuki, Mamoru Watanabe, Toshifumi Hibi

\section{ACKNOWLEDGEMENTS}

The authors would like to thank all CERISIER Trial collaborators, investigators, and patients for their participation and contribution to this study. The authors also thank Dr. Fumiaki Ueno, PhD from the Center for Gastroenterology and Inflammatory Bowel Disease, Ofuna Chuo Hospital, Kamakura, Kanagawa, Japan and Prof. Kitaro Futami, PhD from the Department of Surgery, Fukuoka University Chikushi Hospital, Chikushino City, Fukuoka, Japan for their clinical review of the efficacy and adverse events of this trial as the Data and Safety Monitoring Board. The authors thank the
Japanese Society for Inflammatory Bowel Disease and EA Pharma Co., Ltd. for supporting the implementation system of this study. The authors thank Angela Morben, DVM, ELS, from Edanz Group (www.edanzediting.com/ac), for editing a draft of this manuscript.

\section{Members of CERISIER Study Group}

Satoshi Motoya (Sapporo Kosei General Hospital), Atsuo Maemoto (Sapporo Higashi Tokushukai Hospital), Mikihiro Fujiya (Asahikawa Medical University), Takayuki Matsumoto (Iwate Medical University), Hirotake Sakuraba (Hirosaki University), Hironori Yamamoto (Jichi Medical University), Hideyuki Hiraishi (Dokkyo Medical University), Yasuharu Yakabi (Saitama Medical University), Ryota Hokari (Natinal Defense Medical College), Toshihede Ohmori (Ohmori Toshihide Gastro-intestinal Clinic), Kiyonori Kobayashi (Kitasato University), Reiko Kunisaki (Yokohama City University Medical Center), Takanori Kanai (Keio University), Mamoru Watanabe (Tokyo Medical and Dental University), Tomoo Nakagawa (Chiba University), Toshifumi Ohkusa (The Jikei University Kashiwa Hospital), Katsutoshi Tokushige (Tokyo Women's Medical University), Yasuo Suzuki (Toho University Sakura Medical Center), Toshifumi Hibi (Kitasato University Kitasato Institute Hospital), Tsuneo Fukushima (Matsushima Clinic), Atsushi Yoshida (Ofuna Chuo Hospital), Takayuki Yamamoto (Yokkaichi Hazu Medical Center), Akihiko Ohta (Ieda Hospital), Katsuhiko Nakai (Matsuda Hospital), Koichiro Matsuda (Toyama Prefectural Central Hospital), Tadashi Yokoyama (Yokoyama Hospital, Yokoyama IBD Clinic), Noriko Kamata (Osaka City University), Shiro Nakamura (Hyogo College of Medicine), Kazuhide Higuchi (Osaka Medical College), Hideki Iijima (Osaka University), Fumiko Minami (Daiwa Hospital), Minoru Matsuura (Kyoto University), Akira Andoh (Shiga University), Tomoyuki Tsujikawa (Higashi-Ohmi Medical Center), Yuji Naito (Kyoto Prefectural University of Medicine), Kenji Watanabe (Osaka City General Hospital), Akiko Shiotani (Kawasaki Medical School), Sakiko Hiraoka (Okayama University), Shinji Tanaka (Hiroshima University), Koichi Kurahara (Matsuyama Red Cross Hospital), Kazuhiro Matsueda (Kurashiki Central Hospital), Toshiyuki Matsui (Fukuoka University Chikushi Hospital), Ryuichi Iwakiri (Saga University). 


\section{REFERENCES}

1. Chan HC, Ng SC. Emerging biologics in inflammatory bowel disease. J Gastroenterol 2017;52:141-150.

2. Akobeng AK, Thomas AG. Enteral nutrition for maintenance of remission in Crohn's disease. Cochrane Database Syst Rev 2007;(3):CD005984. doi: 10.1002/14651858.CD005984.pub2.

3. Yamamoto T, Nakahigashi M, Umegae S, Matsumoto K. Enteral nutrition for the maintenance of remission in Crohn's disease: a systematic review. Eur J Gastroenterol Hepatol 2010;22:1-8.

4. Tsertsvadze A, Gurung T, Court R, Clarke A, Sutcliffe P. Clinical effectiveness and cost-effectiveness of elemental nutrition for the maintenance of remission in Crohn's disease: a systematic review and meta-analysis. Health Technol Assess 2015;19:1138.

5. Hirai F, Ishihara H, Yada S, et al. Effectiveness of concomitant enteral nutrition therapy and infliximab for maintenance treatment of Crohn's disease in adults. Dig Dis Sci 2013;58:13291334 .
6. Kamata N, Oshitani N, Watanabe K, et al. Efficacy of concomitant elemental diet therapy in scheduled infliximab therapy in patients with Crohn's disease to prevent loss of response. Dig Dis Sci 2015;60:1382-1388.

7. Sazuka S, Katsuno T, Nakagawa T, et al. Concomitant use of enteral nutrition therapy is associated with sustained response to infliximab in patients with Crohn's disease. Eur J Clin Nutr 2012;66:1219-1223.

8. Sugita N, Watanabe K, Kamata N, et al. Efficacy of a concomitant elemental diet to reduce the loss of response to adalimumab in patients with intractable Crohn's disease. J Gastroenterol Hepatol 2018;33:631-637.

9. Nguyen DL, Palmer LB, Nguyen ET, McClave SA, Martindale RG, Bechtold ML. Specialized enteral nutrition therapy in Crohn's disease patients on maintenance infliximab therapy: a meta-analysis. Therap Adv Gastroenterol 2015;8:168-175.

10. Yamamoto T, Nakahigashi M, Umegae S, Matsumoto K. Prospective clinical trial: enteral nutrition during maintenance infliximab in Crohn's disease. J Gastroenterol 2010;45:24-29. 